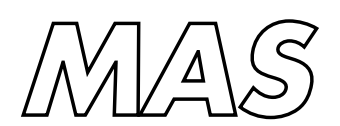

Modelling, Analysis and Simulation

Modelling, Analysis and Simulation
MAS A continuum model of lipid bilayers

J.G. Blom, M.A. Peletier

Report MAS-R0229 NOVember 30, 2002 
CWI is the National Research Institute for Mathematics and Computer Science. It is sponsored by the Netherlands Organization for Scientific Research (NWO).

$\mathrm{CWI}$ is a founding member of ERCIM, the European Research Consortium for Informatics and Mathematics.

CWI's research has a theme-oriented structure and is grouped into four clusters. Listed below are the names of the clusters and in parentheses their acronyms.

Probability, Networks and Algorithms (PNA)

Software Engineering (SEN)

Modelling, Analysis and Simulation (MAS)

Information Systems (INS)

Copyright @ 2001, Stichting Centrum voor Wiskunde en Informatica

P.O. Box 94079, 1090 GB Amsterdam (NL)

Kruislaan 413, 1098 SJ Amsterdam (NL)

Telephone +31205929333

Telefax +31205924199

ISSN 1386-3703 


\title{
A Continuum Model of Lipid Bilayers
}

\author{
J.G. Blom and M.A. Peletier \\ $C W I$
}

P.O. Box 94079, 1090 GB Amsterdam, The Netherlands

\begin{abstract}
We study a one-dimensional continuum model for lipid bilayers. The system consists of water and lipid molecules; lipid molecules are represented by two 'beads', a head bead and a tail bead, connected by a rigid rod. We derive a simplified model for such a system, in which we only take into account the effects of entropy and hydrophilic/hydrophobic interactions. We show that for this simple model membrane-like structures exist for certain choices of the parameters, and numerical calculations suggest that they are stable.
\end{abstract}

2000 Mathematics Subject Classification: Primary: 49K22. Secondary: 74L15, 92C10.

Keywords and Phrases: Partial differential equations, integral equations, biomechanics, lipid bilayers, continuum model.

Note: Work carried out under project MAS1.1 - PDEs in the Life Sciences.

\section{IntRoduction}

Lipid bilayers, biological membranes, are the cell's main separating structure. They shield the interior of the cell from the outside and divide the intracellular space into compartments. In many places the concentration of chemical species differs across the membrane, a fact that is essential to cellular metabolism.

This paper is part of a larger effort to develop a continuum model for lipid bilayers that reproduces a number of aspects of actual bilayers. The most salient of these are the elastic properties: one can bend or stretch a bilayer, and this will result in a counteracting force which is capable of restoring the original geometry upon release. Such behaviour is not commonly found in current reaction-diffusion models, and in this paper we make a suggestion as to how such behaviour might be captured in a continuum setup.

In this paper we study a simplified, one-dimensional reduction of a three-dimensional model. For this one-dimensional model we derive the equations satisfied by solutions (Section 4), discuss the existence of membrane-like structures (Sections 5-6), investigate a semi-explicit solution (Section 7), and present some numerical results (Section 8). For completeness the three-dimensional 'parent' model is presented in Appendix 1.

\section{LIPID BILAYERS}

Lipid bilayers have an interesting physicochemical structure. The main component is a lipid molecule (Fig. 1) which consists of a head and two tails. The head is usually charged and hydrophilic, while the tails are hydrophobic. This difference in water affinity causes lipids to aggregate, and in the biological setting the lipids are typically found in a bilayer structure as shown in Fig. 1. In such a structure the energetically unfavorable tail-water interactions are avoided by grouping the tails together in a water-free zone, shielded from the water by the heads. While for other amphiphilic molecules spherical or linear arrangements are also possible, most lipids have a preference for planar structures.

It is important that there is no chemical bonding between any two lipids. In fact, movement in the directions parallel to the plane is relatively unrestricted. Out-of-plane movement, however, carries an energy penalty, as does removal or breakage, since these changes increase tail-water interaction. It is this energetic effect that ensures the integrity of the bilayer as a planar structure. 


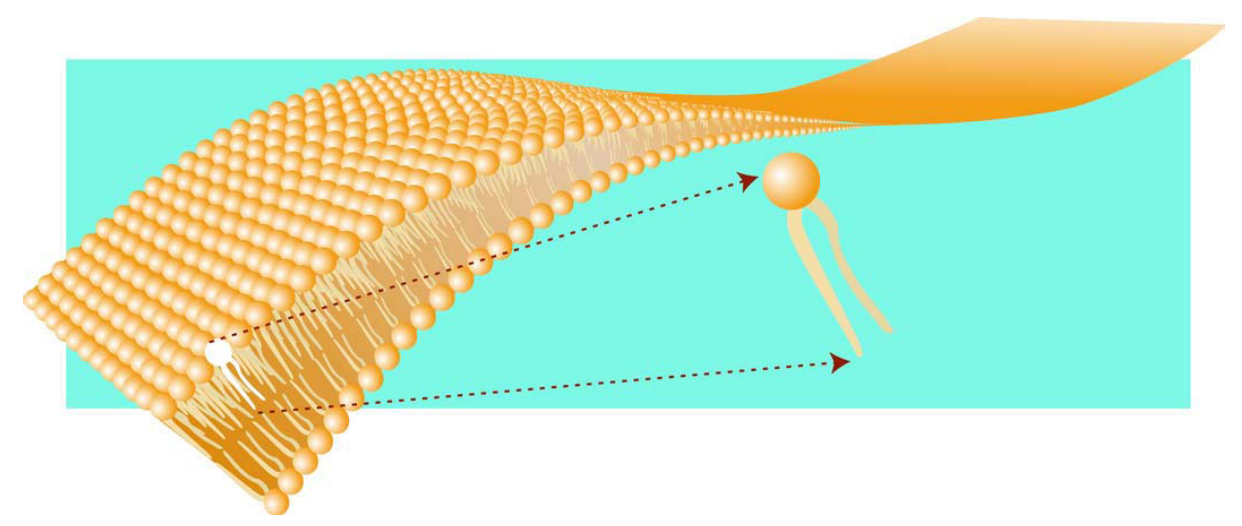

Figure 1: A section of a lipid bilayer

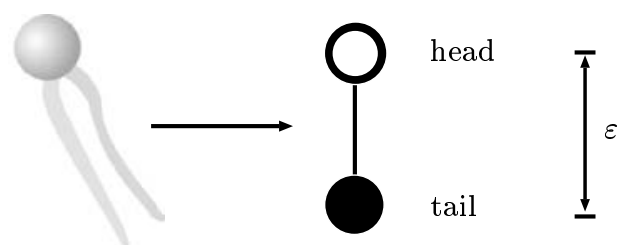

Figure 2: In the simplified model of this paper the lipid molecule is represented by head and tail beads, connected by a rigid rod.

\section{A SIMPLIFIED ONE-DIMENSIONAL MODEL}

The one-dimensional model of this paper is a simplified version of the three-dimensional model derived in Appendix 1. In this model the lipid and water molecules are represented by 'beads': water molecules are single beads, and lipid molecules consist of $t$ wo beads connected by rigid, mass-less rods (Fig. 2).

The single spatial dimension $(x)$ should be thought of as orthogonal to the plane of a membrane. The rods connecting head and tail beads in the lipids are assumed to lie parallel to the $x$-axis; therefore there are $t$ no species of lipids, those with the tail pointing in the positive and those with the tail pointing in the negative $x$-direction. The unknowns $u$ and $v$ are the densities of these $t$ wo species, or to be more precise, the densities of the tail beads of these $t$ no species (Figure 3). If the connecting $\operatorname{rod}$ has length $\varepsilon$, the corresponding head bead volume fractions are the translated fields $\tau_{-\varepsilon} u$ and $\tau_{\varepsilon} v$. The function $w$ is the density of the water beads. We assume that all beads are of equal size, so that the densities $u, v$, and $w$ can also be viewed as volume fractions.

The character of the model is determined by choosing a free-energy functional, which we then seek to minimize. We choose a combination of entropy, compression energy, and interaction energy: ${ }^{1}$

$$
\begin{aligned}
T \int[\eta(u)+\eta(v)+\eta(w)]+\frac{p}{2} \int\left[1-u-v-\tau_{-\varepsilon} u-\tau_{\varepsilon} v-w\right]^{2} & \\
& +\alpha \int\left(w+\tau_{-\varepsilon} u+\tau_{\varepsilon} v\right) \kappa *(u+v),
\end{aligned}
$$

\footnotetext{
${ }^{1}$ The convolution $*$ is defined as

$$
(f * g)(x)=\int f(x-y) g(y) d y .
$$
}



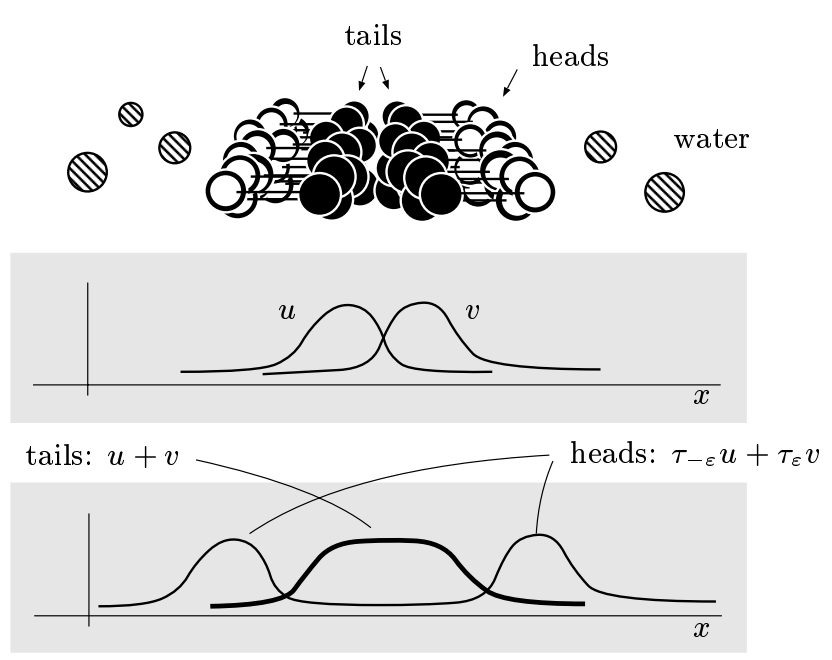

Figure 3: Schematic diagram of a 'membrane'. The graphs show concentrations as a function of $x$, the coordinate orthogonal to the membrane. Water bead concentration is not plotted.

where

$$
\eta(s)= \begin{cases}s \log s & s \geq 0 \\ \infty & s<0 .\end{cases}
$$

The first integral in (3.1) is the Gibbs-Boltzmann entropy, multiplied by temperature $T$. This term is convex and favours spreading of mass. The second integral models the compression of the mixture, since

$$
\underbrace{u(x)+v(x)}_{\text {tails }}+\underbrace{\tau_{-\varepsilon} u(x)+\tau_{\varepsilon} v(x)}_{\text {heads }}+\underbrace{w(x)}_{\text {water }}
$$

is the total number of beads at a given position $x$ (we have scaled densities to make unity the reference density). The constant $p$ characterizes the compressibility. The third integral is non-convex and will be responsible for the appearance of non-constant energy minimizers. It penalizes proximity between the tails (with density $u+v)$ and heads and water $\left(w+\tau_{-\varepsilon} u+\tau_{\varepsilon} v\right)$. The interaction kernel $\kappa$ is a model parameter; for the length of this paper we shall assume that $\kappa$ is given by

$$
\kappa(s)=\frac{1}{2} e^{-|s|} .
$$

This specific choice allows for the detailed analysis performed in Section 7.

In this paper, however, we consider a further simplification of (3.1), by assuming that the material is incompressible $(p=\infty)$. This implies that the aggregate bead count (3.2) is constant (scaled to 1), so that $w$ is related to $u$ and $v$ by

$$
w=1-u-v-\tau_{-\varepsilon} u-\tau_{\varepsilon} v .
$$

This allows us to consider $u$ and $v$ as the unknowns in the model. The non-negativity of $w$ then imposes a constraint on the functions $u$ and $v$ :

$$
u+v+\tau_{-\varepsilon} u+\tau_{\varepsilon} v \leq 1 .
$$


The free energy then reads

$$
\int[\eta(u)+\eta(v)]+\alpha \int(1-u-v) \kappa *(u+v),
$$

where the temperature $T$ has been scaled into the coefficient $\alpha$. Note that in the passage from (3.1) to (3.5) we have discarded the term modelling the entropy of the water molecules. The reason for disregarding the water entropy is to make the system amenable to analysis; we expect that for most modelling questions the effects of this simplification will be small, and this is an issue that we will return to in future.

\section{The Minimization PROBlem}

We now extract a precise mathematical problem from the preceding discussion, and from here on we aim for mathematical rigour. We are concerned with unknowns $(u, v)$ that are functions defined on the real line. Experiments suggest (see Section 5 below) considering functions $u$ and $v$ that converge to a value $0<c_{0}<1 / 4$ at $\pm \infty$ (the limit value has to be the same for $u$ and $v$; cf. (4.6-4.7)). We therefore introduce the class of admissible functions

$$
C=\left\{(u, v) \in\left(c_{0}, c_{0}\right)+L^{1}(\mathbb{R})^{2}: u+\tau_{-\varepsilon} u+v+\tau_{\varepsilon} v \leq 1 \text { a.e. }\right\} .
$$

The constant $c_{0}$ is called a background concentration.

The free energy for this system is

$$
F(u, v)=\int\left[\eta(u)+\eta(v)+\alpha(1-u-v) \kappa *(u+v)-\zeta\left(c_{0}\right)\right]
$$

where the constant $\zeta\left(c_{0}\right)=2 \eta\left(c_{0}\right)+\alpha c_{0}\left(1-2 c_{0}\right)$ is added to ensure integrability.

In the rest of this paper we will be concerned with minimization of the free energy $F$ in the class $C$ with an additional constraint on the mass:

$$
\int\left[u+v-2 c_{0}\right]=m \text {. }
$$

The Euler-Lagrange equations satisfied by a minimizer will involve the Fréchet derivative of $F$; for future reference we first calculate this derivative on its own:

$$
\begin{aligned}
& F^{\prime}(u, v) \cdot(\bar{u}, \bar{v})= \\
& \quad=\int\left[\eta^{\prime}(u) \bar{u}+\eta^{\prime}(v) \bar{v}+\alpha(-\bar{u}-\bar{v}) \kappa *(u+v)+\alpha(1-u-v) \kappa *(\bar{u}+\bar{v})\right] \\
& \quad=\int\left[\eta^{\prime}(u) \bar{u}+\eta^{\prime}(v) \bar{v}+\alpha(\bar{u}+\bar{v}) \kappa *(1-2 u-2 v)\right]
\end{aligned}
$$

Using the identification of $\left[L^{1}(\mathbb{R})^{2}\right]^{\prime}$ with $L^{\infty}(\mathbb{R})^{2}$, and provided $u$ and $v$ are bounded away from zero, we can write

$$
F^{\prime}(u, v)=(\log u+1+\alpha \kappa *(1-2 u-2 v), \log v+1+\alpha \kappa *(1-2 u-2 v))
$$

Theorem 1 (Euler-Lagrange equations). Let $(u, v)$ be a solution of the minimization problem

$$
\min \left\{F(u, v):(u, v) \in C, \quad \int\left(u+v-2 c_{0}\right)=m\right\} .
$$


Then there exist Lagrange multipliers $\lambda \in \mathbb{R}$ and $\mu \in L^{\infty}(\mathbb{R})$ such that

$$
\begin{aligned}
& \log u-2 \alpha \kappa *(u+v)=\lambda-\mu-\tau_{\varepsilon} \mu \\
& \log v-2 \alpha \kappa *(u+v)=\lambda-\mu-\tau_{-\varepsilon} \mu .
\end{aligned}
$$

In addition $\mu \geq 0$ on $\mathbb{R}$, and

$$
\operatorname{supp} \mu \subset \Omega_{s}:=\left\{x \in \mathbb{R}:\left(u+\tau_{-\varepsilon} u+v+\tau_{\varepsilon} v\right)(x)=1\right\}
$$

The set $\Omega_{s}$ is called the saturation set; the word saturation refers to the water volume fraction being zero in $\Omega_{s}$.

Corollary 2. Let the extended saturation set be defined as

$$
\Omega_{e s}=\{-\varepsilon, 0, \varepsilon\}+\Omega_{s}
$$

Then $u=v$ a.e. on the complement of $\Omega_{e s}$.

Proof. On the complement of $\Omega_{e s}$ the right-hand sides of (4.6) and (4.7) both reduce to $\lambda$; therefore $u$ and $v$ coincide.

Proof of Theorem 1. We use the framework of dual convex cones, as detailed in Appendix 2. Therefore we introduce the affine operator

$$
B:\left(c_{0}, c_{0}\right)+L^{1}(\mathbb{R})^{2} \longrightarrow\left[1-4 c_{0}+L^{1}(\mathbb{R})\right] \times \mathbb{R} \times \mathbb{R}
$$

representing the constraints,

$$
B(u, v)=\left(1-u-\tau_{-\varepsilon} u-v-\tau_{\varepsilon} v, \int\left(u+v-2 c_{0}\right)-m,-\int\left(u+v-2 c_{0}\right)+m\right),
$$

such that the set $C^{\prime}$ of admissible functions in (4.5) can be written as

$$
C^{\prime}=\left\{(u, v) \in\left(c_{0}, c_{0}\right)+L^{1}(\mathbb{R})^{2}: B(u, v) \geq 0\right\} .
$$

Let $K \subset L^{1}(\mathbb{R})^{2}$ be the cone of admissible directions of perturbation, i.e.

$$
K=\left\{(\tilde{u}, \tilde{v}) \in L^{1}(\mathbb{R})^{2}: \exists \eta>0 \text { such that }(u, v)+\eta(\tilde{u}, \tilde{v}) \in C^{\prime}\right\} .
$$

Defining the linear operator $b$ as the derivative of $B$,

$$
\begin{aligned}
& b: L^{1}(\mathbb{R})^{2} \longrightarrow L^{1}(\mathbb{R}) \times \mathbb{R} \times \mathbb{R} \\
& b(\tilde{u}, \tilde{v})=\left(-\tilde{u}-\tau_{-\varepsilon} \tilde{u}-\tilde{v}-\tau_{\varepsilon} \tilde{v}, \int(\tilde{u}+\tilde{v}),-\int(\tilde{u}+\tilde{v})\right),
\end{aligned}
$$

we can characterize $K$ differently:

$$
\begin{aligned}
K & =\left\{(\tilde{u}, \tilde{v}) \in L^{1}(\mathbb{R})^{2}: B((u, v)+\eta(\tilde{u}, \tilde{v})) \geq 0 \text { for some } \eta>0\right\} \\
& =\left\{(\tilde{u}, \tilde{v}) \in L^{1}(\mathbb{R})^{2}: B(u, v)+\eta b(\tilde{u}, \tilde{v}) \geq 0 \text { for some } \eta>0\right\} \\
& =\left\{(\tilde{u}, \tilde{v}) \in L^{1}(\mathbb{R})^{2}: b(\tilde{u}, \tilde{v}) \geq-B(u, v) / \eta \text { for some } \eta>0\right\} \\
& =\left\{(\tilde{u}, \tilde{v}) \in L^{1}(\mathbb{R})^{2}: b(\tilde{u}, \tilde{v})_{2}=b(\tilde{u}, \tilde{v})_{3}=0, b(\tilde{u}, \tilde{v})_{1}(x) \geq 0 \text { if } B(u, v)_{1}(x)=0\right\} .
\end{aligned}
$$


Here $b(\tilde{u}, \tilde{v})_{i}$ is the $i^{\text {th }}$ component of $b(\tilde{u}, \tilde{v})$. Note that the condition $B(u, v)_{1}(x)=0$ in this last characterization of $K$ is equivalent to $x \in \Omega_{s}$. Simplifying the description slightly by introducing the bounded linear operator

$$
\begin{aligned}
& A: L^{1}(\mathbb{R})^{2} \longrightarrow L^{1}(\mathbb{R}) \times \mathbb{R} \times \mathbb{R} \\
& A(\tilde{u}, \tilde{v})=\left(\left(-\tilde{u}-\tau_{-\varepsilon} \tilde{u}-\tilde{v}-\tau_{\varepsilon} \tilde{v}\right) \chi_{\Omega_{s}}, \int(\tilde{u}+\tilde{v}),-\int(\tilde{u}+\tilde{v})\right),
\end{aligned}
$$

where $\chi_{\Omega_{s}}$ is the characteristic function of the set $\Omega_{s}$, we can write

$$
K=\left\{(\tilde{u}, \tilde{v}) \in L^{1}(\mathbb{R})^{2}: A(\tilde{u}, \tilde{v}) \geq 0\right\} .
$$

By standard arguments the assumption that $(u, v)$ is a minimizer implies that $F^{\prime}(u, v) \cdot(\tilde{u}, \tilde{v}) \geq 0$ for all $(\tilde{u}, \tilde{v}) \in K$. Therefore $F^{\prime}(u, v) \in K^{\perp}$, and by Lemma 9 this implies that there exist $\left(\mu, \lambda_{+}, \lambda_{-}\right) \in$ $L^{\infty}(\mathbb{R}) \times \mathbb{R} \times \mathbb{R}$, with $\mu, \lambda_{+}, \lambda_{-} \geq 0$, such that

$$
\begin{aligned}
F^{\prime}(u, v) & =A^{T}\left(\mu, \lambda_{+}, \lambda_{-}\right) \\
& =\left(-\mu \chi_{\Omega_{s}}-\tau_{\varepsilon}\left(\mu \chi_{\Omega_{s}}\right)+\lambda_{+}-\lambda_{-},-\mu \chi_{\Omega_{s}}-\tau_{-\varepsilon}\left(\mu \chi_{\Omega_{s}}\right)+\lambda_{+}-\lambda_{-}\right) .
\end{aligned}
$$

Upon redefining $\mu=\mu \chi_{\Omega_{s}}$ and setting $\lambda=\lambda_{+}-\lambda_{-}-1-\alpha$ the statement of the theorem follows.

Remark 3 Theorem 1 only refers to minimizers, not to stationary points. Following the proof of Theorem 1, we define a constrained stationary point of $F$ as a pair $(u, v) \in C^{\prime}$ with $F^{\prime}(u, v) \cdot(\tilde{u}, \tilde{v})=0$ for all $(\tilde{u}, \tilde{v}) \in K$. Then the assertion of Theorem 1 applies, and in addition $\mu \equiv 0$.

\section{The Critical Micelle Concentration}

A well-known feature of amphiphile molecules, such as lipids, is the existence of a critical micelle concentration (CMC; see e.g. (author?) [4, Chapter 1]). If a mixture of lipids and water is prepared with lipid concentration less than the CMC, then all lipids are present in monomer form, i.e. no aggregates are formed. If one attempts to exceed the CMC, however, then it is observed that the monomer concentration does not increase, but the surplus lipids aggregate into larger structures such as micelles and planar membranes.

In this section we investigate this feature in the simplified model derived above, and we show that indeed such a behaviour can be recognized in this model. We emulate the experiment in this model in the following way. Starting with a vessel filled with uniform volume fraction $\left(c_{0}, c_{0}\right)$, we add a quantity $m$ of lipids, i.e. we consider elements $(u, v)$ of the set

$$
C^{\prime \prime}=\left\{(u, v) \in C: u \geq c_{0}, v \geq c_{0}, \int\left(u+v-2 c_{0}\right)=m\right\} .
$$

We investigate the 'response of the system' by considering sequences in $C^{\prime \prime}$ that minimize the free energy $F$, i.e. sequences $\left(u_{n}, v_{n}\right) \in C^{\prime \prime}$ satisfying

$$
\lim _{n \rightarrow \infty} F\left(u_{n}, v_{n}\right)=\inf _{C^{\prime \prime}} F .
$$

We want to distinguish between aggregation and non-aggregation. We say that a sequence $\left(u_{n}, v_{n}\right) \in$ $C^{\prime \prime}$ aggregates if there exist $L>0$ and $x_{n} \in \mathbb{R}$ such that

$$
\liminf _{n \rightarrow \infty} \int_{x_{n}-L}^{x_{n}+L}\left(u_{n}+v_{n}-2 c_{0}\right)>0
$$


On the other hand we say that a sequence spreads when no finite region remains different from the background concentration:

$$
\lim _{n \rightarrow \infty} \sup _{x \in \mathbb{R}} \int_{x-L}^{x+L}\left(u_{n}+v_{n}-2 c_{0}\right)=0 \quad \text { for all } L>0 .
$$

Note that the supremum over $x$ is necessary to avoid applying the term 'spreading' to an aggregate that moves away to infinity.

There is a small middle ground between the two definitions: it is possible that a subsequence spreads, so that (5.1) is violated, and that a different subsequence aggregates, violating (5.2). It is true, however, that a sequence that does not aggregate contains a spreading subseqence, and vice versa.

Our first result states that if we choose the background volume fraction $c_{0}$ sufficiently small, then no aggregate configuration can minimize the energy, and every minimizing sequence spreads:

Theorem 4. Fix $\alpha, \varepsilon>0$. There exists $0<c_{c} \leq 1 / 4$ such that for all $0<c_{0}<c_{c}$ and for all $m>0$ every minimizing sequence $\left(u_{n}, v_{n}\right) \in C^{\prime \prime}$ spreads.

On the other hand, for a given background volume fraction $c_{0}$ we can force the system to aggregate by choosing $\alpha$ sufficiently large:

Theorem 5. Fix $\varepsilon>0$ and $0<c_{0}<1 / 4$. There exist $\alpha_{c}>0$ and $m_{c}>0$ such that for all $\alpha>\alpha_{c}$ and all $m>m_{c}$ every minimizing sequence aggregates.

Theorem 5 suggests that minimizing sequences have some form of compactness in $L^{1}(\mathbb{R})^{2}$, and the numerical experiments that we present below confirm this suggestion. We hope to return to this issue in a later publication.

Remark 6 The definition of $C^{\prime \prime}$ requires the 'surplus mass' functions $u-c_{0}$ and $v-c_{0}$ to be nonnegative. Without this requirement the minimization problem $\inf _{C^{\prime \prime}} F$ may not be well-posed. This can be seen by taking the specific sequence

$$
u_{n}(x)=c_{0}+n^{-1} \operatorname{sech}\left(n^{-4} x\right) \sin \left(n^{-2} x\right), \quad v_{n} \equiv c_{0},
$$

for which $m=0$ and

$$
F\left(u_{n}, v_{n}\right) \sim\left(\eta^{\prime \prime}\left(c_{0}\right)-2 \alpha\right) n^{2} \quad \text { as } \quad n \rightarrow \infty .
$$

If $\eta^{\prime \prime}\left(c_{0}\right)-2 \alpha<0$, then $F$ is not bounded from below on $C^{\prime \prime}$.

\section{Proofs of Theorems 4 And 5}

Lemma 7. Let $\left(u_{n}, v_{n}\right) \in C^{\prime \prime}$ be a spreading sequence. Then

$$
\liminf _{n \rightarrow \infty} F\left(u_{n}, v_{n}\right) \geq m\left(\eta^{\prime}\left(c_{0}\right)+\alpha\left(1-4 c_{0}\right)\right) .
$$

Proof of Lemma \%. Set $\left(u_{n}, v_{n}\right)=\left(c_{0}+\bar{u}_{n}, c_{0}+\bar{v}_{n}\right)$, such that $\int(\bar{u}+\bar{v})=m$. Using the identity $\int \kappa * u=\int u$ we write the functional $F$ as

$$
F(u, v)=\int\left[\eta(u)+\eta(v)+\alpha(u+v)-\zeta\left(c_{0}\right)\right]-\alpha \int(u+v) \kappa *(u+v),
$$


so that

$$
\begin{aligned}
& F\left(u_{n}, v_{n}\right)-m\left(\eta^{\prime}\left(c_{0}\right)+\alpha\left(1-4 c_{0}\right)\right)= \\
& =\int\left[\eta\left(c_{0}+\bar{u}_{n}\right)-\eta\left(c_{0}\right)-\eta^{\prime}\left(c_{0}\right) \bar{u}_{n}\right]+\int\left[\eta\left(c_{0}+\bar{v}_{n}\right)-\eta\left(c_{0}\right)-\eta^{\prime}\left(c_{0}\right) \bar{v}_{n}\right] \\
& \quad+\alpha \int\left[\left(1-2 c_{0}-\bar{u}_{n}-\bar{v}_{n}\right) \kappa *\left(2 c_{0}+\bar{u}_{n}+\bar{v}_{n}\right)-\left(1-2 c_{0}\right)\left(2 c_{0}\right)-\left(1-4 c_{0}\right)\left(\bar{u}_{n}+\bar{v}_{n}\right)\right] \\
& \quad-\alpha \int\left(\bar{u}_{n}+\bar{v}_{n}\right) \kappa *\left(\bar{u}_{n}+\bar{v}_{n}\right) . \\
& \left.\quad \int\left[\eta\left(c_{0}+\bar{u}_{n}\right)-\eta\left(c_{0}\right)-\eta^{\prime}\left(c_{0}\right) \bar{u}_{n}\right]+\int\left[c_{0}+\bar{v}_{n}\right)-\eta\left(c_{0}\right)-\eta^{\prime}\left(c_{0}\right) \bar{v}_{n}\right] \\
& \quad
\end{aligned}
$$

The first two terms are non-negative by the convexity of $\eta$, and we only need to show that the last term vanishes.

Set $w_{n}=\bar{u}_{n}+\bar{v}_{n} \geq 0$ and define

$$
\xi(L, n)=\sup _{y \in \mathbb{R}} \int_{y-L}^{y+L} w_{n},
$$

so that for each $L>0, \xi(L, n) \rightarrow 0$ as $n \rightarrow \infty$. Since $\kappa$ is symmetric and decreasing away from zero, and pointwise bounded by $1 / 2$,

$$
\begin{aligned}
\int w_{n} \kappa * w_{n} & =w_{n} \int \kappa(y) w_{n}(x-y) w_{n}(x) d x d y \\
& \leq \frac{1}{2} \int_{|y| \leq L} \int_{\mathbb{R}} w_{n}(x-y) w_{n}(x) d x d y+\kappa(L) \int_{|y| \geq L} \int_{\mathbb{R}} w_{n}(x-y) w_{n}(x) d x d y \\
& \leq \frac{1}{2} \xi(L, n) \int w_{n}+\kappa(L)\left(\int w_{n}\right)^{2} \\
& \longrightarrow 0 \quad \text { as } n, L \longrightarrow \infty
\end{aligned}
$$

Proof of Theorem 4. Lemma 7 gives a lower bound for the energy of a spreading sequence, and by choosing functions of the form

$$
u_{n}(x)=c_{0}+n^{-1} f\left(x n^{-1}\right), \quad v_{n}(x)=c_{0}+n^{-1} f\left(x n^{-1}\right),
$$

where $2 \int f=m$, one shows that this lower bound is sharp:

$$
\lim _{n \rightarrow \infty} F\left(u_{n}, v_{n}\right)=m\left(\eta^{\prime}\left(c_{0}\right)+\alpha\left(1-4 c_{0}\right)\right) .
$$

We now show that this limit value is optimal when $c_{0}$ is small. For a given admissible pair $(u, v)$, we set $(u, v)=\left(c_{0}+\bar{u}, c_{0}+\bar{v}\right)$, such that $\int(\bar{u}+\bar{v})=m$. Then

$$
\begin{aligned}
& F(u, v)-m\left[\eta^{\prime}\left(c_{0}\right)+\alpha\left(1-4 c_{0}\right)\right]= \\
& =\int\left[\eta\left(c_{0}+\bar{u}\right)-\eta\left(c_{0}\right)-\eta^{\prime}\left(c_{0}\right) \bar{u}\right]+\int\left[\eta\left(c_{0}+\bar{v}\right)-\eta\left(c_{0}\right)-\eta^{\prime}\left(c_{0}\right) \bar{v}\right] \\
& \quad-\alpha \int(\bar{u}+\bar{v}) \kappa *(\bar{u}+\bar{v})
\end{aligned}
$$


Using the estimate

$$
\int f \kappa * f \leq \int f^{2}
$$

we find

$$
\begin{aligned}
& F(u, v)-m\left[\eta^{\prime}\left(c_{0}\right)+\alpha\left(1-4 c_{0}\right)\right] \geq \\
& \geq \int\left[\eta\left(c_{0}+\bar{u}\right)-\eta\left(c_{0}\right)-\eta^{\prime}\left(c_{0}\right) \bar{u}\right]+\int\left[\eta\left(c_{0}+\bar{v}\right)-\eta\left(c_{0}\right)-\eta^{\prime}\left(c_{0}\right) \bar{v}\right] \\
& \quad-\alpha \int(\bar{u}+\bar{v})^{2} \\
& \quad \geq \int(h(\bar{u})+h(\bar{v})),
\end{aligned}
$$

where $h$ is given by

$$
h(\bar{u})=\eta\left(c_{0}+\bar{u}\right)-\eta\left(c_{0}\right)-\eta^{\prime}\left(c_{0}\right) \bar{u}-2 \alpha \bar{u}^{2} .
$$

Using $\eta^{\prime \prime}(0+)=\infty$ it is easily verified that if $c_{0}>0$ is sufficiently small, then there exists $\beta>0$ such that

$$
h(\bar{u}) \geq \beta \bar{u}^{2} \quad \text { for all } 0 \leq \bar{u} \leq 1 .
$$

Therefore

$$
F(u, v)-m\left[\eta^{\prime}\left(c_{0}\right)+\alpha\left(1-4 c_{0}\right)\right] \geq \beta \int\left[\left(u-c_{0}\right)^{2}+\left(v-c_{0}\right)^{2}\right] .
$$

It follows that if $\left(u_{n}, v_{n}\right) \in C^{\prime \prime}$ is a minimizing sequence, then $\left(u_{n}-c_{0}, v_{n}-c_{0}\right) \rightarrow 0$ in $L^{2}(\mathbb{R})^{2}$. The spreading (5.2) then follows from the inequality

$$
\int_{x-L}^{x+L}\left|u_{n}+v_{n}-2 c_{0}\right| \leq \sqrt{2 L}\left(\int_{x-L}^{x+L}\left(u_{n}+v_{n}-2 c_{0}\right)^{2}\right)^{1 / 2} .
$$

Proof of Theorem 5. As we saw above the energy of a spreading sequence is equal to

$$
m\left[\eta^{\prime}\left(c_{0}\right)+\alpha\left(1-4 c_{0}\right)\right] .
$$

Below we exhibit a function $(u, v)$ with energy $F(u, v)$ less than this value (say $F_{0}$ ). This proves Theorem 5 by the following argument. Minimizing sequences must have limit energy values less than or equal to $F_{0}$, and therefore strictly less than (6.1). If the sequence does not aggregate, then there exists a subsequence that spreads; along this subsequence the energy converges to a value larger than or equal to (6.1). This is a contradiction.

Set $m_{c}=\varepsilon\left(1-2 c_{0}\right)$, and assume that $m_{c} \leq m \leq 2 m_{c}$. We now construct a function $(u, v)$ with energy less than (6.1). Set

$$
u(x)=v(x)= \begin{cases}\frac{1}{2} & \text { if } 0<x<\frac{m}{1-2 c_{0}}, \\ c_{0} & \text { otherwise }\end{cases}
$$


Then $\int\left(u-c_{0}\right)=\int\left(v-c_{0}\right)=m / 2$ and $u+\tau_{-\varepsilon} u+v+\tau_{\varepsilon} v \leq 1$, so that $(u, v) \in C^{\prime \prime}$. Again writing $(\bar{u}, \bar{v})=\left(u-c_{0}, v-c_{0}\right)$, we have

$$
\begin{aligned}
& F(u, v)- m\left[\eta^{\prime}\left(c_{0}\right)+\alpha\left(1-4 c_{0}\right)\right]= \\
&=\int\left[\eta\left(c_{0}+\bar{u}\right)-\eta\left(c_{0}\right)-\eta^{\prime}\left(c_{0}\right) \bar{u}\right]+\int\left[\eta\left(c_{0}+\bar{v}\right)-\eta\left(c_{0}\right)-\eta^{\prime}\left(c_{0}\right) \bar{v}\right] \\
& \quad-\alpha \int(\bar{u}+\bar{v}) \kappa *(\bar{u}+\bar{v}) \\
&=2 \frac{m}{1-2 c_{0}}\left[\eta(1 / 2)-\eta\left(c_{0}\right)-\eta^{\prime}\left(c_{0}\right)\left(1 / 2-c_{0}\right)\right]-4 \alpha \int \bar{u} \kappa * \bar{u} .
\end{aligned}
$$

Using the explicit calculation

$$
\int \chi_{[0, \ell]} \kappa * \chi_{[0, \ell]}=e^{-\ell}-1+\ell
$$

where $\chi_{A}$ is the characteristic function of the set $A$, we have

$$
\begin{aligned}
F(u, v)- & m\left[\eta^{\prime}\left(c_{0}\right)+\alpha\left(1-4 c_{0}\right)\right]= \\
= & \frac{4 m}{1-2 c_{0}}\left[\eta(1 / 2)-\eta\left(c_{0}\right)-\eta^{\prime}\left(c_{0}\right)\left(1 / 2-c_{0}\right)\right] \\
& \quad-\alpha\left(1-2 c_{0}\right)^{2}\left[\exp \left(-\frac{m}{1-2 c_{0}}\right)-1+\frac{m}{1-2 c_{0}}\right] .
\end{aligned}
$$

We can now choose $\alpha_{c}>0$ such that the right-hand side is negative for all $\alpha>\alpha_{c}$ and for all $m_{c} \leq m \leq 2 m_{c}$.

For values of $m$ larger than $m_{c}$, we split the total mass $m$ into $n$ parts of size in $\left[m_{c} / 2, m_{c}\right]$. For each part $i=1, \ldots, n$, we construct a function $\left(u_{i}, v_{i}\right)$ of the type $(6.2)$; we assemble these in the following way: choose numbers $\ell_{i}, i=1, \ldots, n$, with $\ell_{i+1}-\ell_{i}>2 \varepsilon+m_{c} /\left(1-2 c_{0}\right)$. Then the function $(u, v)$ is given by

$$
(u, v)(x)=\left(c_{0}, c_{0}\right)+\sum_{i=1}^{n}\left[\left(u_{i}, v_{i}\right)\left(x-\ell_{i}\right)-\left(c_{0}, c_{0}\right)\right] .
$$

The function $F$ is additive with respect to this type of addition, and we therefore conclude that

$$
F(u, v)-m\left[\eta^{\prime}\left(c_{0}\right)+\alpha\left(1-4 c_{0}\right)\right]<0,
$$

whenever $\alpha>\alpha_{c}$ (which is given above). This proves the assertion.

\section{A (NEARLY) EXPLICIT SOLUTION}

A stationary point $(u, v)$ which is such that the pointwise inequality (4.1) is not saturated anywhere on $\mathbb{R}$ solves the Euler-Lagrange equations (see Remark 3)

$$
\begin{aligned}
& \log u-2 \alpha \kappa *(u+v)=\lambda, \\
& \log v-2 \alpha \kappa *(u+v)=\lambda .
\end{aligned}
$$

Therefore $u \equiv v$, and the two Euler-Lagrange equations for $u$ and $v$ reduce to a single equation,

$$
\log u-4 \alpha \kappa * u=\lambda .
$$

Using the value at infinity we determine that $\lambda$ is equal to $\log c_{0}-4 \alpha c_{0}$, so that (7.1) can be written as

$$
\log \frac{u}{c_{0}}=4 \alpha \kappa *\left(u-c_{0}\right) .
$$


Set $\phi=\kappa *\left(u-c_{0}\right)$, so that

$$
-\phi^{\prime \prime}+\phi=u-c_{0} \quad \text { on } \mathbb{R} \text {. }
$$

Combining (7.2) and (7.3) we find

$$
-\phi^{\prime \prime}+\phi=c_{0}\left(e^{4 \alpha \phi}-1\right) \quad \text { on } \mathbb{R} \text {. }
$$

This equation can be integrated to obtain

$$
\frac{1}{2}{\phi^{\prime}}^{2}+G(\phi)=0
$$

with

$$
G(\phi)=-\frac{1}{2} \phi^{2}+\frac{c_{0}}{4 \alpha}\left(e^{4 \alpha \phi}-1-4 \alpha \phi\right) .
$$

The value of the constant of integration has been determined by requiring that solutions converge to zero at infinity. For this equation to have such a solution it is necessary that zero be a local maximum of $G$, or

$$
\alpha c_{0}<\frac{1}{4}
$$

In this case, the aspect of $G$ is as shown in Figure 4 . At the bifurcation point $\alpha c_{0}=1 / 4$ the minimum in this figure coalesces with the local maximum at zero, leaving the function $G$ monotonic.

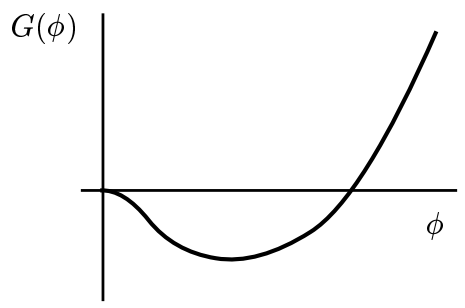

Figure 4: The form of the potential $G$ for $\alpha c_{0}<1 / 4$.

Figure 5 shows the dependency of the additional mass that is contained in such a solution. At the point marked with a circle the constraint is first violated.

We now turn briefly to the stability of this solution. Figure 5 shows that the mass of a solution decreases as $\alpha$ increases. For a stable solution this is surprising: we would expect the mass in a global minimizer to increase when we increase the destabilizing effect of the energy term multiplied by $\alpha$.

On the other hand, this parameter dependence is exactly what one expects for a saddle point connecting two valleys, a mountain pass. We therefore conjecture that this solution is of the mountain pass type.

\section{NumericAl RESUlts}

In addition to the analytical results given above we present a first numerical result. We seek local minima of $F$ under constraints, i.e. solutions of the minimization problem (4.5). We implemented a constrained $L^{2}$-gradient flow algorithm for the free energy functional $F$, under the constraint of constant mass (4.3). The constraint of positive water concentration was implemented by adding a penalization term $(K / 2) \int\left(u+\tau_{-\varepsilon} u+v+\tau_{\varepsilon} v-1\right)_{+}^{2}$ to $F$. 


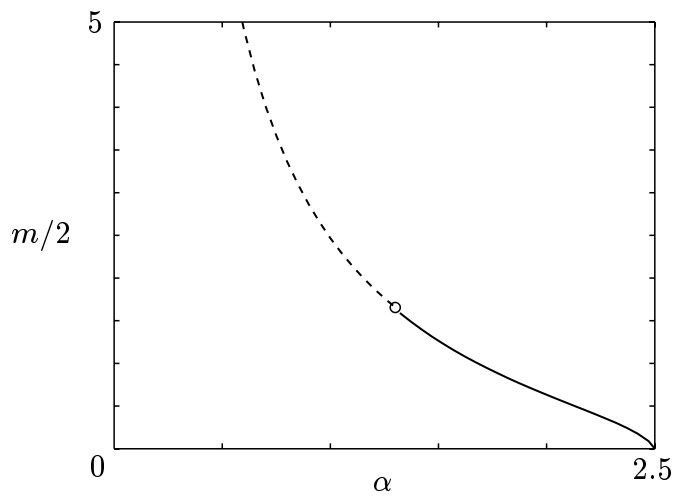

Figure 5: The curve of semi-explicit solutions given by (7.4). The solid line indicates solutions satifying the pointwise constraint (4.3), the dotted line solutions that violate this constraint. Here $c_{0}=0.1$ and $\varepsilon=1$.

The resulting equations are

$$
\begin{aligned}
& u_{t}=-\log u+2 \alpha \kappa *(u+v)+\lambda-\mu-\tau_{\varepsilon} \mu, \\
& v_{t}=-\log v+2 \alpha \kappa *(u+v)+\lambda-\mu-\tau_{-\varepsilon} \mu,
\end{aligned}
$$

where $\lambda$ is a Lagrange multiplier and $\mu$ is given by

$$
\mu=K\left(u+\tau_{-\varepsilon} u+v+\tau_{\varepsilon} v-1\right)_{+} .
$$

For the numerical approximation a finite domain $[-L, L]$ was chosen. On such a finite domain the 'background concentration' $c_{0}$ loses its special status; for instance, under constraint of given total mass on $[-L, L]$ the value of $u+v$ at the boundaries $x= \pm L$ is free to change (and does change). (For the alternative choice, Dirichlet boundary conditions on $u$ and $v$, the mass is not conserved. Here we expect that stable single membrane-solutions do not exist; if aggregation is more favorable, energy-wise, than spreading, then the aggregate will draw in mass from the boundary until the whole domain is filled with aggregate).

For the calculation of the convolution $\kappa *(u+v)$ the $u+v$ was considered to be constant on $[L, \infty)$ (and equal to the value at $x=L$ ).

The functions $u$ and $v$ were discretized in space on a uniform grid; the convolution integral was approximated by the midpoint rule. The resulting system of differential-algebraic equations was solved by an implicit ODE solver.

Figure 6 shows two examples of 'membranes' that were found in this way.

\section{Conclusions}

There is a clear rationale for pushing the continuum description of living systems as far as possible. Continuum models have proven both to be convenient objects for theoretical study, thereby giving an important type of theoretical insight into the underlying system, and to provide an efficient framework for numerical simulation.

In the case of living cells the representation of chemical reactions in a continuum framework is well understood. The mechanical side of cellular function, however, is much less well developed; although models are available for various parts of the cellular machinery, integration of such descriptions into a complete continuum formulation is not yet feasible.

It is one of the challenges for such a continuum formulation to capture the mechanical behaviour of lipid bilayers. In this paper we have shown that a simple, one-dimensional model is capable of 


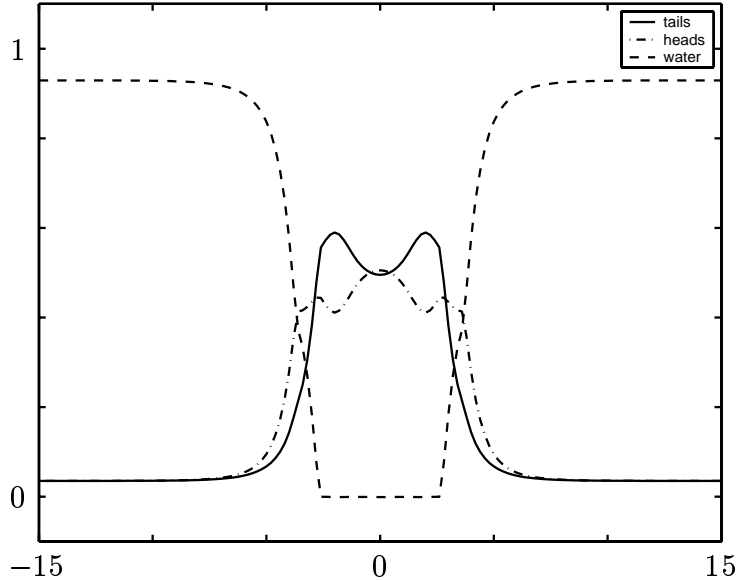

(a) $\varepsilon=1$

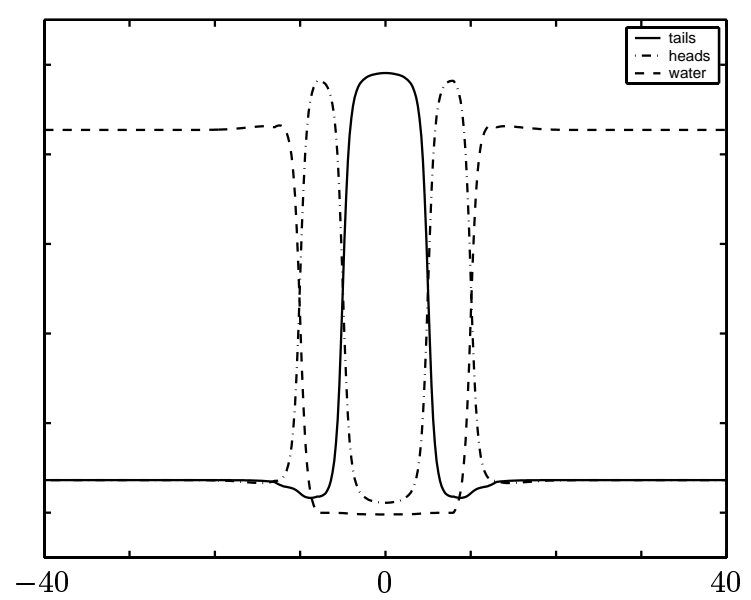

(b) $\varepsilon=5$

Figure 6: Stationary 'single-membrane solutions'. Plotted are the concentrations of tails $(u+v)$, heads $\left(\tau_{-\varepsilon} u+\tau_{\varepsilon} v\right)$, and water $\left(1-u-\tau_{-\varepsilon} u-v-\tau_{\varepsilon} v\right)$. Parameters were $\alpha=4, \varepsilon \in\{1,5\}, L=100$, and $K=1000$. For both choices of $\varepsilon$ the structure is well localized, but for larger $\varepsilon$ the internal separation of heads and tails is much better developed. Note the saturation zone (zero water concentration) in the middle of the membrane.

reproducing a minimal set of properties: for certain choices of the parameters membrane-like structures exist and are (at least numerically) locally stable. In addition, the occurrence or non-occurrence of membrane-like structures depends on the background concentration $c_{0}$ in a way that is consistent with experiments.

This paper is only the first step, however. The one-dimensionality of this model, the restriction to rod-like lipid molecules, the restriction to two-bead molecules, the hydrophilic/-phobic interaction between heads, tails, and water, and many more choices that have been made each raise questions about the effects of relaxing such assumptions. In addition a more thorough investigation of the model of this paper is necessary to fully understand the effects of the various parameters.

\section{ThE THREE-DIMENSIONAL MODEL}

The description that we use leans heavily on the 'mesoscopic dynamics' framework introduced by Fraaije and his coworkers (author?) [2, 3].

For the derivation of the model we start at a microscopic, sub-continuum scale. We consider a vessel, represented by $\Omega \subset \mathbb{R}^{3}$, containing $N_{w}$ water molecules and $N_{\ell}$ lipid molecules. Each lipid molecule is represented by a collection of $n$ connected 'beads'; water molecules consist of a single bead. The variable $X_{w}^{j}$, represents the position of water molecule $j, 1 \leq j \leq N_{w}$, and takes values in $\Omega$. The position of bead $i$ of lipid $j, 1 \leq i \leq n, 1 \leq j \leq N_{\ell}$, is $X_{\ell}^{i, j}$, and the set of bead positions of a single lipid molecule $\left(X_{\ell}^{1, j}, \ldots, X_{\ell}^{n, j}\right)$ takes values in a subset $V \subset \Omega^{n}$. In many cases $V$ will simply coincide with $\Omega^{n}$; however, for the important class of models with rod-like links between the beads, $V$ is a strict subset of $\Omega^{n}$. The full state space for the system is therefore

$$
\mathcal{X}=\Omega^{N_{w}} \times V^{N_{\ell}} .
$$

Elements $X=\left(X_{w}^{1}, \ldots, X_{w}^{N_{w}}, X_{\ell}^{1,1}, \ldots, X_{\ell}^{n, 1}, X_{\ell}^{1,2}, \ldots, X_{\ell}^{n, N_{\ell}}\right) \in \mathcal{X}$ are called microstates.

We describe the system in terms of probabilities on $\mathcal{X}$, i.e. the (probabilistic) state $\psi$ is a probability 
measure on $\mathcal{X}$ :

$$
\psi \in \mathcal{E}, \quad \text { where } \quad \mathcal{E}=\left\{\psi: \mathcal{X} \rightarrow \mathbb{R}^{+}, \quad \int_{\mathcal{X}} \psi=1\right\} .
$$

We assume that neither the microstates themselves nor the measure $\psi$ can be observed at the continuum level; the observables are three derived quantities, the volume fractions of water, of heads, and of tails. 'Heads' and 'tails' refer to a colouring of the beads of the lipids: we choose sets $I_{h}, I_{t} \subset\{1, \ldots, n\}$ as those beads that belong to the head(s) or to the tail(s). For simplicity we assume that all beads are either heads or tails, or $I_{h} \cup I_{t}=\{1, \ldots, n\}$.

For a given probability measure $\psi \in \mathcal{E}$, the water volume fraction $r_{w}(\psi): \Omega \rightarrow \mathbb{R}^{+}$is defined by

$$
r_{w}(\psi)(x)=v_{w} \sum_{j=1}^{N_{w}} \int_{\mathcal{X}} \psi \delta\left(X_{w}^{j}-x\right) d X \quad \text { for all } x \in \Omega .
$$

Here $v_{w}$ is the volume fraction of a single water bead. Similarly, we define the head and tail volume fraction by

$$
\begin{array}{ll}
r_{h}(\psi)(x)=v_{h} \sum_{j=1}^{N_{w}} \sum_{i \in I_{h}} \int_{\mathcal{X}} \psi \delta\left(X_{\ell}^{i, j}-x\right) d X, & x \in \Omega \\
r_{t}(\psi)(x)=v_{t} \sum_{j=1}^{N_{w}} \sum_{i \in I_{t}} \int_{\mathcal{X}} \psi \delta\left(X_{\ell}^{i, j}-x\right) d X, & x \in \Omega .
\end{array}
$$

To specify the behaviour of the system we introduce two free energy functionals. The 'ideal' free energy $F^{\text {id }}: \mathcal{E} \rightarrow \mathbb{R}$ models the effects of entropy and the interactions between beads of a single molecule; the 'non-ideal' free energy $F^{\text {nid }}: \mathcal{E} \rightarrow \mathbb{R}$ represents the interactions between the beads of different molecules. The total free energy is the sum of the two,

$$
F(\psi)=F^{\mathrm{id}}(\psi)+F^{\mathrm{nid}}(\psi)
$$

For $F^{\text {id }}$ we postulate

$$
F^{\mathrm{id}}(\psi)=k T \int_{\mathcal{X}} \psi \log \psi+\int_{\mathcal{X}} \psi H^{\mathrm{id}}
$$

The first term is the Boltzmann-Gibbs entropy. In the second term the function $H^{\text {id }}: \mathcal{X} \rightarrow \mathbb{R}$ is the internal energy of a microstate, where the superscript 'id' again refers to a restriction to interaction within a single molecule. We assume that $H^{\text {id }}$ has the form

$$
H^{\mathrm{id}}(X)=\sum_{j=1}^{N_{\ell}} h\left(X_{\ell}^{1, j}, \ldots, X_{\ell}^{n, j}\right)
$$

The choice of $h$ defines the interactions within a single lipid molecule, and we expect this choice to have a significant influence on the properties of the model. We therefore briefly list some examples:

1. Two beads with a rigid link: $n=2$, and $V$ is a true subset of $\Omega^{2}$ :

$$
V=\left\{\left(X^{1}, X^{2}\right) \in \Omega^{2}:\left|X^{1}-X^{2}\right|=\varepsilon\right\},
$$

where $\varepsilon$ is the length of the link. Since deformation is not possible in this setup, $h \equiv 0$. The main body of this paper is based on this lipid structure. 
2. Two beads with a linear spring link: $n=2, V=\Omega^{2}$, and

$$
h\left(X^{1}, X^{2}\right)=\frac{k}{2}\left[\left|X^{1}-X^{2}\right|-\varepsilon\right]^{2}
$$

where $\varepsilon \geq 0$ is the neutral length of the spring.

3. A linear chain of beads connected by springs: Here $V=\Omega^{n}$, and

$$
h\left(X^{1}, \ldots, X^{n}\right)=\sum_{i=1}^{n-1} \frac{k_{i}}{2}\left[\left|X^{i}-X^{i+1}\right|-\varepsilon_{i}\right]^{2} .
$$

Obviously many other variations are possible.

For the non-ideal free energy $F^{\text {nid }}$ we make an important simplifying assumption: $F^{\text {nid }}$ can be written as a function of only the observables,

$$
F^{\text {nid }}(\psi)=F^{\text {nid }}\left(r_{w}(\psi), r_{h}(\psi), r_{t}(\psi)\right)
$$

Typical terms in the non-ideal energy $F^{\text {nid }}$ are a convolution integral, in which proximity of hydrophilic (heads and water) beads and hydrophobic tail beads is penalized:

$$
\int_{\Omega} \int_{\Omega}\left(r_{w}(\psi)(x)+r_{h}(\psi)(x)\right) r_{t}(\psi)(y) \kappa(x-y) d x d y
$$

and a compressibility term that penalizes deviation from unit total volume:

$$
\frac{1}{2} \int_{\Omega}\left(r_{w}(\psi)(x)+r_{t}(\psi)(x)+r_{h}(\psi)(x)-1\right)^{2} d x
$$

We now turn to the continuum scale. Our unknowns at this scale are the volume fractions of water, of heads, and of tails; we denote these by $\rho_{w}, \rho_{h}$, and $\rho_{t}$. Note the difference with the previously introduced $r_{w}, r_{h}$, and $r_{t}$ : the former are the unknowns in the continuum problem, the latter are operators that map probability measures in $\mathcal{E}$ to volume fractions on $\Omega$. However, if $\psi$ is the microscopic probabilistic state belonging to the continuum state $\left(\rho_{w}, \rho_{h}, \rho_{t}\right)$, then the two coincide:

$$
\rho_{w, h, t}(x)=r_{w, h, t}(\psi)(x) \quad \text { for all } x \in \Omega .
$$

For the unknowns $\left(\rho_{w}, \rho_{h}, \rho_{t}\right)$ we postulate the evolution equations

$$
\frac{\partial \rho_{\alpha}}{\partial t}=\operatorname{div}\left[\rho_{\alpha} \nabla\left(U_{\alpha}+\frac{\partial}{\partial \rho_{\alpha}} F^{\mathrm{nid}}\right)\right], \quad \alpha=w, h, t .
$$

Here we identify the Fréchet derivative $\partial / \partial \rho_{\alpha} F^{\text {nid }}$ with its $L^{2}$-gradient, i.e. $\partial / \partial \rho_{h} F^{\text {nid }}$ should be interpreted as the function $v \in L^{2}(\Omega)$ satisfying

$$
\int_{\Omega} v \phi=\frac{\partial}{\partial \rho_{h}} F^{\text {nid }}\left(\rho_{h}, \rho_{t}, \rho_{w}\right) \cdot \phi \quad \text { for all } \phi \in L^{2}(\Omega) .
$$

The chemical potential $U_{\alpha}$ is determined by solving the minimization problem

$$
\min \left\{F^{\mathrm{id}}(\psi): \psi \in \mathcal{E}, r_{w, h, t}(\psi)=\rho_{w, h, t}\right\} .
$$

The Euler-Lagrange equation for the minimizer $\psi$ reads

$$
\left(F^{\mathrm{id}}\right)^{\prime}(\psi) \cdot \tilde{\psi}-\lambda \int_{\mathcal{X}} \tilde{\psi}(X) d X-\sum_{\alpha=w, h, t} \int_{\Omega} U_{\alpha}(x) r_{\alpha}(\tilde{\psi})(x) d x=0
$$


for any $\tilde{\psi}: \mathcal{X} \rightarrow \mathbb{R}$. The chemical potentials $U_{\alpha}: \Omega \rightarrow \mathbb{R}$ arise as Lagrange multipliers associated with the constraints on $r_{\alpha}$. Since $F^{\text {id }}$ is smooth and strictly convex, the mapping

$$
\left(\rho_{w}, \rho_{h}, \rho_{t}\right) \mapsto\left(U_{w}, U_{h}, U_{t}\right)
$$

is well-defined and smooth.

The interpretation of this setup is as follows. There is a separation of time scales: changes in the microstate that conserve the observables $r_{w, h, t}$ happen on a shorter time scale than changes in the observables themselves. At the observable time scale we therefore assume that the system is in equilibrium with respect to the fast degrees of freedom. This is achieved by minimizing the free energy under constraint, as in (1.2).

Remark 8 The evolution equations (1.1) can be viewed as the gradient flow of the free energy $F$ with respect to a metric of the Wasserstein type in the three unknowns $\rho_{w}, \rho_{h}, \rho_{t}$ :

$$
g_{\rho_{\alpha}}\left(\frac{\partial \rho_{\alpha}}{\partial t}, s\right)=-\frac{\partial F}{\partial \rho_{\alpha}} \cdot s \quad \forall s, \forall \alpha=w, h, t
$$

where $g_{\rho_{\alpha}}(\cdot, \cdot)$ is the Wasserstein metric tensor (e.g. (author?) [5]).

At this stage the model derivation proper is complete; the resulting system is expected to be wellposed in a reasonable sense (we will return to this question in a future publication). However, we can simplify the description considerably without losing generality.

Written in full the Euler-Lagrange equation (1.3) reads

$$
\begin{aligned}
& k T \int_{\mathcal{X}}(\log \psi+1) \tilde{\psi}+\int_{\mathcal{X}} \tilde{\psi} H^{\mathrm{id}}-\lambda \int_{\mathcal{X}} \tilde{\psi}-v_{w} \sum_{j=1}^{N_{w}} \int_{\mathcal{X}} U_{w}\left(X_{w}^{j}\right) \tilde{\psi}(X) d X \\
& \quad-v_{h} \sum_{j=1}^{N_{\ell}} \sum_{i \in I_{h}} \int_{\mathcal{X}} U_{h}\left(X_{\ell}^{i, j}\right) \tilde{\psi}(X) d X-v_{t} \sum_{j=1}^{N_{\ell}} \sum_{i \in I_{t}} \int_{\mathcal{X}} U_{t}\left(X_{\ell}^{i, j}\right) \tilde{\psi}(X) d X=0,
\end{aligned}
$$

for all $\psi \in L^{1}(\mathcal{X})$, implying that

$$
\begin{aligned}
\psi(X)=C \exp \left\{\frac { 1 } { k T } \left[-H^{\mathrm{id}}(X)+v_{w} \sum_{j=1}^{N_{w}} U_{w}(\right.\right. & \left.X_{w}^{j}\right) \\
& \left.\left.\quad+v_{h} \sum_{j=1}^{N_{\ell}} \sum_{i \in I_{h}} U_{h}\left(X_{\ell}^{i, j}\right)+v_{t} \sum_{j=1}^{N_{\ell}} \sum_{i \in I_{t}} U_{t}\left(X_{\ell}^{i, j}\right)\right]\right\},
\end{aligned}
$$

where $C$ is a normalization constant. We can therefore decompose $\psi$ as

$$
\psi(X)=\prod_{j=1}^{N_{w}} \psi_{w}\left(X_{w}^{j}\right) \prod_{j=1}^{N_{\ell}} \psi_{\ell}\left(X_{\ell}^{1, j}, \ldots, X_{\ell}^{n, j}\right) .
$$

The microscopic state function $\psi$ has been replaced by two single-molecule state functions $\psi_{w}$ and $\psi_{\ell}$, which satisfy

$$
\begin{aligned}
\psi_{w}(x) & =C_{w} \exp \left\{v_{w} U_{w}(x) / k T\right\} \\
\psi_{\ell}\left(X^{1}, \ldots, X^{n}\right) & =C_{\ell} \exp \left\{\frac{1}{k T}\left[-h\left(X^{1}, \ldots, X^{n}\right)+v_{h} \sum_{i \in I_{h}} U_{h}\left(X^{i}\right)+v_{t} \sum_{i \in I_{t}} U_{t}\left(X^{i}\right)\right]\right\} .
\end{aligned}
$$


It follows that

$$
\begin{aligned}
r_{w}(\psi)(x) & =v_{w} N_{w} \psi_{w}(x) \\
r_{h, t}(\psi)(x) & =v_{h, t} N_{\ell} \sum_{i \in I_{h, t}} \int_{V} \psi_{\ell}\left(X^{1}, \ldots, X^{n}\right) \delta\left(x-X^{i}\right)
\end{aligned}
$$

Using this description we can rework the equations (1.1-1.2-1.3) into a more convenient form, by eliminating the microscopic state $\psi$. The equations (1.1) remain the same:

$$
\frac{\partial \rho_{\alpha}}{\partial t}=\operatorname{div}\left[\rho_{\alpha} \nabla\left(U_{\alpha}+\frac{\partial}{\partial \rho_{\alpha}} F^{\mathrm{nid}}\right)\right], \quad \alpha=w, h, t,
$$

but we now specify the relationship between $\rho_{\alpha}$ and $U_{\alpha}$ directly. For water,

$$
\frac{1}{v_{w} N_{w}} \rho_{w}=C_{w} \exp \left\{v_{w} U_{w}(x) / k T\right\}, \quad \text { with } \quad C_{w}^{-1}=\int_{\Omega} \exp \left\{v_{w} U_{w}(x) / k T\right\}
$$

For heads and tails, the equations are

$$
\begin{aligned}
& \frac{1}{v_{h, t} N_{\ell}} \rho_{h, t}=C_{\ell} \int_{V}\left[\sum_{i \in I_{h, t}} \delta\left(x-X^{i}\right)\right] \times \\
& \exp \left\{\frac{1}{k T}\left[-h\left(X^{1}, \ldots, X^{n}\right)+v_{h} \sum_{i \in I_{h}} U_{h}\left(X^{i}\right)+v_{t} \sum_{i \in I_{t}} U_{t}\left(X^{i}\right)\right]\right\}
\end{aligned}
$$

where again

$$
C_{\ell}^{-1}=\int_{V} \exp \left\{\frac{1}{k T}\left[-h\left(X^{1}, \ldots, X^{n}\right)+v_{h} \sum_{i \in I_{h}} U_{h}\left(X^{i}\right)+v_{t} \sum_{i \in I_{t}} U_{t}\left(X^{i}\right)\right]\right\} .
$$

When supplemented with a definition of $F^{\text {nid }}$, the equations $(1.6-1.9)$ constitute a closed system.

The equation for water can be simplified even further. From (1.7) it follows that

$$
\nabla \rho_{w}(t, x)=\frac{v_{w}}{k T} \rho_{w}(t, x) \nabla U_{w}(t, x)
$$

so that equation (1.6) for $\rho_{w}$ can be written as

$$
\frac{\partial}{\partial t} \rho_{w}=\frac{k T}{v_{w}} \Delta \rho_{w}+\operatorname{div}\left[\rho_{w} \nabla\left(\frac{\partial}{\partial \rho_{w}} F^{\text {nid }}\right)\right]
$$

Note that, as demonstrated by this equation, the effect of the entropy in the ideal free energy is to include a diffusive term in the equations.

\section{CONES AND DUALITY}

In this appendix we briefly recall some facts on duality cones.

Let $X$ be a Banach space, and let $\mathcal{P} \subset X$ be a closed convex cone. The dual cone $\mathcal{P}^{\perp} \subset X^{\prime}$ is defined as

$$
\mathcal{P}^{\perp}=\left\{f \in X^{\prime}:\langle f, u\rangle \geq 0 \quad \forall u \in \mathcal{P}\right\},
$$

and is again closed and convex. 
We now introduce a second Banach space $Y$, and a bounded linear operator $A: Y \rightarrow X$. Let $K$ be the following cone in $Y$ :

$$
K=\{u \in Y: A u \in \mathcal{P}\}
$$

and its dual is by definition

$$
K^{\perp}=\left\{f \in Y^{\prime}:\langle f, u\rangle \geq 0 \quad \forall u \in K\right\} .
$$

Our main result is a characterization of the dual cone $K^{\perp}$ :

Lemma 9. $K^{\perp}=\left\{A^{T} g \in Y^{\prime}: g \in \mathcal{P}^{\perp}\right\}$.

Note that Lemma 9 does not require $X$ or $Y$ to be reflexive; therefore some arguments need careful attention. We use the following result:

Lemma 10. If $C$ is a closed convex cone in a Banach space $X$, then $C^{\perp \perp} \cap X=C$.

Proof of Lemma 10. First the inclusion $C \subset C^{\perp \perp} \cap X$ : if $u \in C$, then

$$
\forall f \in C^{\perp}, \quad\langle f, u\rangle \geq 0 .
$$

Therefore $u \in C^{\perp \perp}$.

$C^{\perp \perp} \cap X \subset C$ : suppose that $u \in C^{\perp \perp} \cap X, u \notin C$. By the Hahn-Banach theorem (e.g. (author?) [1]) there exists $f \in C^{\perp}$ such that $\langle f, u\rangle<0$. But this contradicts the assumption that $u \in C^{\perp \perp}$.

Proof of Lemma 9. Set $M=\left\{A^{T} g \in Y^{\prime}: g \in \mathcal{P}^{\perp}\right\}$.

$M \subset K^{\perp}$ : let $A^{T} g \in M$, implying that $g \in \mathcal{P}^{\perp}$. Then

$$
\forall u \in K, \quad\left\langle A^{T} g, u\right\rangle=\langle g, A u\rangle \geq 0 .
$$

Therefore $A^{T} g \in K^{\perp}$.

$K^{\perp} \subset M$ : we first show that $M^{\perp} \subset K$. If $u \in M^{\perp}$, then by definition

$$
\langle f, u\rangle \geq 0 \quad \text { for all } f \in M,
$$

so that

$$
\begin{array}{ll} 
& \left\langle A^{T} g, u\right\rangle \geq 0 \quad \text { for all } g \in \mathcal{P}^{\perp} \\
\Longrightarrow & \langle g, A u\rangle \geq 0 \quad \text { for all } g \in \mathcal{P}^{\perp} \\
\Longrightarrow & A u \in \mathcal{P}^{\perp \perp} \cap X=\mathcal{P},
\end{array}
$$

implying that $u \in K$, and therefore that $M^{\perp} \subset K$. By Lemma 10 it now follows that $K^{\perp} \subset$ $M^{\perp \perp} \cap Y^{\prime}=M$.

\section{ACKNowledgement}

The authors are grateful to Georg Prokert for his insightful comments on an early version of the manuscript. This research was supported by the RTN network 'Nonlinear Partial Differential Equations describing Front Propagation and other Singular Phenomena', HPRN-CT-2002-00274. 


\section{Bibliography}

[1] H. Brezis. Analyse fonctionnelle. Masson, Paris, 1983.

[2] J. G. E. M. Fraaije. Dynamic density functional theory for microphase separation kinetics of block copolymer melts. J. Chem. Phys., 99:9202-9212, 1993.

[3] J. G. E. M. Fraaije, B. A. C. van Vlimmeren, N. M. Maurits, M. Postma, O. A. Evers, C. Hoffmann, P. Altevogt, and G. Goldbeck-Wood. The dynamic mean-field density functional method and its application to the mesoscopic dynamics of quenched block copolymer melts. J. Chem. Phys., 106:4260-4269, 1997.

[4] W. M. Gelbart, A. Ben-Shaul, and D. Roux, editors. Micelles, Membranes, Microemulsions and Monolayers. Springer-Verlag, New York, 1995.

[5] F. Otto. The geometry of dissipative evolution equations: the porous medium equation. Comm. PDE, 26:101-174, 2001. 